\title{
DFS pattern and DFS70 autoantibody testing: comments on the article "Investigation of anti-DFS70 antibody in patients with systemic autoimmune rheumatic diseases"
}

\author{
Chuiwen Deng ${ }^{1}$ (D) \\ Received: 23 December 2019 /Revised: 23 December 2019 / Accepted: 10 January 2020 / Published online: 13 March 2020
}

(C) International League of Associations for Rheumatology (ILAR) 2020

I read with great interest the article published by Peker et al. (Clinical Rheumatology (2019) 38:3627-3633.) [1]. It is interesting that their conclusion was similar to the observation we have published prior [2]. Currently, verification of the nuclear dense fine speckled (DFS) pattern, also known as the AC-2 pattern, by a specific anti-DFS70 assay is considered essential to exclude the pseudo-DFS pattern [3]. Peker et al. did confirm the DFS pattern by the other kit. However, the enzyme-linked immunosorbent assay (ELISA) kit they used is designed to detect the DFS70 antigen in serum. Until now, no report shows that the levels of DFS70 antigen are related with DFS70 autoantibodies, and no published studies confirmed the DFS pattern by evaluating the levels of DFS70 antigen. Moreover, Peker et al. planned to use an autoantibody testing kit for they mention in the article that DFS patterns were confirmed by specific DFS70 antibody ELISA kits.

See related article, https://doi.org/10.1007/s10067-019-04730-y

Chuiwen Deng

chuiwen21@163.com

1 Departments of Rheumatology and Clinical Immunology Center, Peking Union Medical College Hospital, Chinese Academy of Medical Science \& Peking Union Medical College, No. 1 Shuaifuyuan, Dongdan, Beijing 100730, China

\section{Compliance with ethical standards}

Disclosures None.

\section{References}

1. Peker B.O., Șener A.G., Tarhan E.F., Kaya S. Investigation of antiDFS70 antibody in patients with systemic autoimmune rheumatic diseases. Clinical Rheumatology 2019 38:12 (3627-3633). https:// doi.org/10.1007/s10067-019-04730-y.

2. Deng C., Qu X., Cheng S., Zeng X., Li Y., Fei Y. Decision-making value of nuclear dense fine speckled pattern in systemic autoimmune rheumatic disease: trick or treat? Ann Rheum Dis. 2019 May 15. pii: annrheumdis-2019-215587. https://doi.org/10.1136/annrheumdis2019-215587

3. Infantino M., Bizzaro N., Grossi V., Manfredi M. The long-awaited 'pseudo-DFS pattern'. Expert Review of Clinical Immunology 2019 15:5 (445). https://doi.org/10.1080/1744666X.2019.1596801

Publisher's note Springer Nature remains neutral with regard to jurisdictional claims in published maps and institutional affiliations. 